\title{
Sustained Effect of Delayed-Release Dimethyl Fumarate in Newly Diagnosed Patients with Relapsing-Remitting Multiple Sclerosis: 6-Year Interim Results From an Extension of the DEFINE and CONFIRM Studies
}

Ralf Gold · Gavin Giovannoni · J. Theodore Phillips · Robert J. Fox •

Annie Zhang · Jing L. Marantz

Received: December 18, 2015 / Published online: March 1, 2016

(C) The Author(s) 2016. This article is published with open access at Springerlink.com

\section{ABSTRACT}

Introduction: Delayed-release dimethyl fumarate (DMF; also known as gastro-resistant DMF) demonstrated clinical and neuroradiologic efficacy and safety in the Phase 3 DEFINE and CONFIRM trials, and in the extension study (ENDORSE), in patients with relapsing-remitting multiple sclerosis (RRMS). This post hoc analysis

Enhanced content To view enhanced content for this article go to www.medengine.com/Redeem/B644F0600 2856AED.

R. Gold

St. Josef Hospital, Ruhr University, Bochum,

Germany

G. Giovannoni

Queen Mary University, London, Blizard Institute, Barts and the London School of Medicine and

Dentistry, London, UK

J. T. Phillips

Multiple Sclerosis Program, Baylor Institute for Immunology Research, Dallas, TX, USA

R. J. Fox

Mellen Center for Multiple Sclerosis Treatment and Research, Cleveland Clinic, Cleveland, OH, USA

A. Zhang · J. L. Marantz ( $₫)$

Biogen, Cambridge, MA, USA

e-mail: jing.marantz@biogen.com assessed DMF efficacy in newly diagnosed patients with RRMS with 6-year minimum follow-up.

Methods: Patients randomized in DEFINE/ CONFIRM to DMF $240 \mathrm{mg}$ twice (BID) or thrice daily (TID) continued on same dosage in ENDORSE. Patients randomized to placebo (PBO) or glatiramer acetate (CONFIRM only) were re-randomized to DMF BID or TID. Results for DMF BID (approved dosage) are reported. Newly diagnosed patients were diagnosed within 1 year prior to DEFINE/CONFIRM entry and either treatment-naive or previously treated with corticosteroids alone.

Results: The newly diagnosed population included 144 patients continuously treated with DMF BID in DEFINE/CONFIRM and ENDORSE (DMF/DMF) and 85 treated with PBO for 2 years in DEFINE/CONFIRM followed by 4 years of DMF BID in ENDORSE (PBO/DMF). At 6 years (ENDORSE Year 4), the annualized relapse rates [ARR; 95\% confidence interval (CI)] were $0.137(0.101,0.186)$ and $0.168(0.113$, 0.252) for DMF/DMF and PBO/DMF, respectively; representing $19 \%$ risk reduction ( $P=0.3988)$. PBO/DMF patients demonstrated improvements in ARR after switching to DMF in ENDORSE: $0.260(0.182,0.372)$ for Years 0-2 
(DEFINE/CONFIRM) and $0.102(0.064,0.163)$ for Years 3-6 (ENDORSE), representing 61\% risk reduction for Years 3-6 versus Years 1-2 $(P<0.0001)$. The proportion of patients with 24 -week confirmed disability progression (95\% $\mathrm{CI})$ at 6 years was $15.7 \%(10.3 \%, 23.7 \%)$ in $\mathrm{DMF} / \mathrm{DMF}$ and $24.3 \%(15.9 \%, 36.2 \%)$ in $\mathrm{PBO} /$ DMF, representing 49\% risk reduction versus $\mathrm{PBO} / \mathrm{DMF}(P=0.0397)$.

Conclusion: Long-term DMF treatment demonstrated strong and sustained efficacy in newly diagnosed patients. Results suggest greater clinical benefits with earlier initiation of treatment in this patient population.

Funding: Biogen.

Trial registration: ClinicalTrials.gov identifiers, NCT00835770 (ENDORSE); NCT00420212 (DEFINE); NCT00451451 (CONFIRM).

Keywords: Delayed-release dimethyl fumarate; Efficacy; Multiple sclerosis; Newly diagnosed; Safety

\section{INTRODUCTION}

Multiple sclerosis (MS) is an inflammatory, demyelinating, neurodegenerative disease affecting the central nervous system [1-3]. More than 2 million people worldwide are affected by this disease, with more than two-thirds of these patients suffering from the relapsing-remitting MS (RRMS) form of the disease $[4,5]$. Patients with relapsing forms of MS experience sporadic relapses that are typically associated with neurologic impairment, disability, and a decrease in overall health and quality of life $[5,6]$. There is extensive variability in the frequency, duration, and severity of symptoms, as well as the extent of recovery [5]. MS begins with the formation of acute inflammatory lesions. Such lesions are often clinically 'silent' and have been estimated to be about 10 times more frequent than episodes of clinical worsening $[7,8]$. This subclinical tissue damage can be visualized by magnetic resonance imaging (MRI). Early in the disease process, the inflammatory activity eventually becomes clinically manifested as a clinically isolated syndrome (CIS) - the first episode of clinically apparent neurologic episodes.

The degenerative processes associated with the progression of the disease include axonal loss in lesions, diffuse damage to white matter distant from areas shown to be involved by histopathology or MRI, and atrophy of deep and cortical grey matter. The later stages of relapsing MS are associated with the accumulation of neuronal loss and gliosis [7]. Therefore, initiating treatment early in the course of relapsing MS could potentially slow disease progression. In fact, clinical trials with interferon $\beta$ and glatiramer acetate (GA) have shown that early treatment was associated with improved outcomes, including a prolonged time to conversion from CIS to clinically definite MS (CDMS) and a reduction in the number and volume of lesions detected by MRI [9-16].

Delayed-release dimethyl fumarate (DMF; also known as gastro-resistant DMF) is a novel, oral MS therapeutic approved for the treatment of patients with relapsing forms of MS. Treatment with DMF has been shown in 2 pivotal Phase 3 trials (DEFINE and CONFIRM) to result in significant reductions in clinical and MRI activity and have a favorable benefit-risk profile in patients with RRMS $[17,18]$. In a post hoc analysis of integrated data from DEFINE and CONFIRM, DMF demonstrated strong efficacy across a broad range of clinical and neuroradiologic outcome measures in patients newly diagnosed with RRMS [19]. Throughout a 
2-year period, DMF $240 \mathrm{mg}$ twice (BID) and thrice daily (TID) resulted in reduced annualized relapse rate (ARR), risk of relapse, proportion of newly diagnosed patients with 12-week confirmed disability progression, odds of having more gadolinium-enhancing $(\mathrm{Gd}+)$ lesions, mean number of new or enlarging T2-hyperintense lesions, and mean number of new non-enhancing T1-hypointense lesions compared with placebo (PBO).

ENDORSE is an ongoing, 8-year extension study of DEFINE and CONFIRM that is being conducted to evaluate the long-term safety and efficacy of DMF in patients with RRMS. The purpose of this paper is to report 6-year clinical efficacy by integrating data from DEFINE, CONFIRM and ENDORSE, to investigate the long-term efficacy of DMF in newly diagnosed patients with RRMS. In addition, summary safety of DMF was also assessed.

\section{METHODS}

\section{Patients and Study Design}

ENDORSE (ClinicalTrials.gov identifier: NCT00835770) is a 2-phase extension study of the DEFINE (ClinicalTrials.gov identifier: NCT00420212) [17] and CONFIRM (ClinicalTrials.gov identifier: NCT00451451) [18] Phase 3 studies, with a total of 10 years of planned follow-up (2 years in the parent studies, DEFINE and CONFIRM, plus 8 years extension in ENDORSE). Further details have been previously reported [19].

DEFINE and CONFIRM included patients 18-55 years of age with RRMS confirmed using McDonald [20] diagnostic criteria. Eligible individuals must also have an Expanded Disability Status Scale (EDSS) [21] score of 0-5.0, inclusive and evidence of disease activity (i.e., relapsed 1 or more times during the year prior to randomization with a prior brain MRI demonstrating 1 or more lesions consistent with MS, or 1 or more Gd+ lesions detected by brain MRI within 6 weeks of randomization). Key exclusion criteria included relapse or corticosteroid treatment within 50 days prior to randomization or prior treatment with GA within 3 months prior to randomization (DEFINE) or at any time (CONFIRM).

In ENDORSE, patients were eligible to enroll if they had participated in and completed, as per protocol, 1 of the 2-year parent studies. Patients were excluded from participating in ENDORSE if there had been any significant change in medical history; if the patient discontinued oral study treatment in the parent studies due to an adverse event (AE) or other reason (except protocol-defined relapse/ disability progression); or if alanine transaminase, aspartate aminotransferase, or gamma-glutamyl transpeptidase increased to greater than 3 times the upper limit of normal.

The ENDORSE extension study was initiated as a multicenter, parallel-group, randomized, dose-blind, dose-comparison study. Patients were enrolled in ENDORSE at Week 96 (last visit of the parent study), which served as the baseline visit for the extension study. In the first phase of ENDORSE, patients who received $240 \mathrm{mg}$ DMF BID or TID in either parent study remained on their same DMF dosage. Patients who received PBO (DEFINE and CONFIRM) or GA (CONFIRM) were re-randomized $1: 1$ to $240 \mathrm{mg}$ DMF BID or TID. Patients were followed every 4 weeks for the first 24 weeks of ENDORSE and every 12 weeks thereafter for up to 8 years. Subsequent to the initiation of ENDORSE, DMF was approved in several countries for the treatment of MS at a dose of $240 \mathrm{mg}$ BID. Effective with the approval, the 
ENDORSE protocol (March 2014) was amended, initiating the second phase. In the second phase, participants receiving DMF $240 \mathrm{mg}$ TID were switched to DMF $240 \mathrm{mg}$ BID dosing at their next scheduled visit.

\section{Efficacy Assessments}

The primary efficacy endpoints were the proportion of patients relapsed at 2 years in DEFINE and ARR at 2 years in CONFIRM. Additional efficacy endpoints included 2-year assessment of time to 12-week confirmed disability progression and numbers of $\mathrm{Gd}+$, new or enlarging T2-hyperintense, and new T1-hypointense lesions. Neurologic exams occurred every 12 weeks for efficacy assessments and at the time of suspected relapse. Relapses were defined as new or recurrent neurologic symptoms, not associated with fever or infection, lasting at least $24 \mathrm{~h}$ and accompanied by new objective neurologic findings. Relapses were confirmed by an Independent Neurologic Evaluation Committee. MRI scans were obtained at baseline and at Weeks 24, 48, and 96. The primary objective of ENDORSE was to evaluate the long-term safety profile of DMF in patients with RRMS. Long-term efficacy outcomes (e.g., ARR, 24-week confirmed EDSS progression) were considered secondary objectives.

Patients diagnosed with RRMS per McDonald diagnostic criteria [20] within 1 year prior to entry into DEFINE and CONFIRM and were either treatment-naïve or previously treated with corticosteroids alone comprised the newly diagnosed population. Prior to the analysis being conducted, the 1-year criterion was chosen because it is the median time since diagnosis of RRMS in the overall treatment-naïve population. Clinical efficacy endpoints were evaluated in post hoc analyses and included ARR and disability progression based on the EDSS score, which was measured every 6 months.

\section{Statistical Analysis}

Integrated data from DEFINE, CONFIRM, and ENDORSE were used in this post hoc analysis. This report, based on the 6-year interim analysis conducted April 15, 2015, presents the long-term efficacy of DMF using clinical endpoints, and it was based on patients who received 1 or more doses of DMF in ENDORSE and had 1 or more post-baseline assessments of the efficacy parameter being analyzed [intent-to-treat (ITT) population]. The analyses were generally based on all observed data prior to switching patients to alternative MS therapies. Clinical efficacy results are summarized for Years 1 and 2 of the parent studies (DEFINE and CONFIRM) and Years 1, 2, 3 , and 4 for the cohort of patients who participated in the ENDORSE extension study. Data are presented according to treatment received in the parent and extension studies. Our analysis focused on DMF BID, as this represents the approved dosage. Patients who received GA were excluded from the analysis since DEFINE did not include a GA-comparator arm and CONFIRM was not designed to compare DMF with GA.

ARR was defined as the total number of relapses divided by the number of patient-years in the study. Data (excluding any collected after patients were switched to alternative MS medications) was analyzed using a negative binomial regression model adjusted for baseline age ( $<40$ vs. $\geq 40$ years), number of relapses in the year prior to study entry, baseline EDSS score $(\leq 2.0$ vs. $>2.0)$, and region pre-defined based on geography, type of health care system, and access to health care 
( $1=$ United States; $2=$ Western Europe, Canada, Costa Rica, Australia, New Zealand, Israel, and South Africa; or 3 = Eastern Europe, India, Guatemala, and Mexico). Disability as measured by time to 24-week confirmed EDSS progression was analyzed using a Cox proportional hazards model, adjusted for the following covariates: baseline EDSS score $\quad(\leq 2.0$ vs. $>2.0)$, baseline age ( $<40$ vs. $\geq 40$ years), region, and number of relapses in the year prior to study entry.

Analyses were performed using SAS version 9.3 software (SAS Institute Inc., Cary, NC, USA).

\section{Compliance with Ethics Guidelines}

All procedures followed were in accordance with the ethical standards of the responsible committee on human experimentation (institutional and national) and with the Helsinki Declaration of 1964, as revised in 2013. Informed consent was obtained from all patients for being included in the study.

\section{RESULTS}

\section{Study Population}

The DEFINE and CONFIRM study populations included 444 patients treated with $\mathrm{PBO}$ or DMF $240 \mathrm{mg}$ BID who met the criteria for newly diagnosed, of whom 362 completed the parent study (189 PBO and 173 DMF) [19]. Of these patients, 229 entered the ENDORSE extension study and were included in the present analysis: 144 continued on DMF 240 mg BID treatment throughout the duration of DEFINE/CONFIRM and ENDORSE (DMF/DMF) and 85 received $\mathrm{PBO}$ in DEFINE/CONFIRM for 2 years and then switched to DMF $240 \mathrm{mg}$ BID in ENDORSE (PBO/DMF; Table 1). Of these patients, 106
DMF/DMF patients and $62 \mathrm{PBO} / \mathrm{DMF}$ patients were female. There was a median (range) follow-up duration of 75.0 (23.0-97.0) months in DMF/DMF patients and 75.0 (14.0-97.3) months in $\mathrm{PBO} / \mathrm{DMF}$ patients. All treatment groups had a mean (standard deviation) time since diagnosis of $0.5(0.5)$ years. In the $\mathrm{PBO} /$ DMF and DMF/DMF groups, $7.1 \%$ and $9.7 \%$ of patients, respectively, received prior steroid treatment. DMF/DMF patients remaining on the study received $\geq 6$ years of continuous DMF treatment, while $\mathrm{PBO} / \mathrm{DMF}$ patients received 2 years of PBO (DEFINE/CONFIRM) followed by $\geq 4$ years of DMF (ENDORSE).

\section{Clinical Efficacy}

In the newly diagnosed population, DMF treatment significantly reduced the frequency of relapse. Over the 6-year duration, including DEFINE/CONFIRM and the ENDORSE extension, the cumulative ARR was numerically lower in patients who received continuous BID treatment (DMF/DMF) than in those who received delayed treatment $(\mathrm{PBO} /$ DMF); cumulative ARRs [95\% confidence interval (CI)] were $0.137(0.101,0.186)$ and $0.168(0.113,0.252)$ for DMF/DMF and PBO/ DMF patients, respectively (Fig. 1a). The rate ratio $(95 \% \mathrm{CI})$ was $0.81(0.51,1.31)$, corresponding with a $19 \%$ risk reduction $(P=0.3988)$. Patients who received delayed treatment (PBO/DMF) demonstrated improvements after switching to DMF in ENDORSE (Fig. 1b). The ARR (95\% CI) for PBO/ DMF patients, from Years 0-2 (DEFINE/ CONFIRM; while on PBO) was 0.260 (0.182, 0.372), which then decreased to 0.102 (0.064, 0.163 ) after switching to DMF in ENDORSE and receiving treatment throughout the next 4 years (rate ratio: 0.39; 95\% CI: 0.24, 0.63). This 
Table 1 Baseline demographic and disease characteristics of the newly diagnosed population at the start of DEFINE and CONFIRM

\begin{tabular}{lll}
\hline Characteristic $^{\mathbf{a}}$ & $\mathbf{D M F}^{\mathbf{b}} / \mathbf{D M F}^{\mathbf{b}}(\boldsymbol{n}=\mathbf{1 4 4})$ & $\mathbf{P B O} / \mathbf{D M F} \mathbf{F}^{\mathbf{b}}(\boldsymbol{n}=\mathbf{8 5})$ \\
\hline Age, years & $35.5(9.2)$ & $36.7(9.1)$ \\
Female (\%) & $106(73.6)$ & $62(72.9)$ \\
Time since first MS symptoms (years) & $4.6(6.2)$ & $4.6(5.4)$ \\
Median (min, max) & $2.0(0.0,42.0)$ & $2.0(0.0,31.0)$ \\
Time since diagnosis (years) & $0.5(0.5)$ & $0.5(0.5)$ \\
Median (min, max) & $0.0(0.0,1.0)$ & $1.0(0.0,1.0)$ \\
Patients with prior treatment with corticosteroids, $n(\%)$ & $14(9.7)$ & $6(7.1)$ \\
Relapses in prior year & $1.4(0.6)$ & $1.3(0.5)$ \\
EDSS score & $2.1(1.2)$ & $2.2(1.0)$ \\
Gd+ lesion volume $\left(\mathrm{cm}^{3}\right)^{\mathrm{c}}$ & $0.4(1.1)$ & $0.2(0.3)$ \\
T2-hyperintense lesion volume $\left(\mathrm{cm}^{3}\right)^{\mathrm{c}}$ & $8.5(9.5)$ & $7.0(6.2)$ \\
T1-hypointense lesion volume $\left(\mathrm{cm}^{3}\right)^{\mathrm{c}}$ & $2.5(3.7)$ & $1.8(2.0)$ \\
\hline
\end{tabular}

EDSS Expanded Disability Status Scale, $G d+$ gadolinium-enhancing, $M R I$ magnetic resonance image, $M S$ multiple sclerosis, $P B O$ placebo

${ }^{a}$ Values are mean (standard deviation) unless otherwise stated

${ }^{b} D M F$ delayed-release dimethyl fumarate (also known as gastro-resistant DMF)

${ }^{c}$ MRI cohort only

represented a $61 \%$ risk reduction for Years 3-6 versus Years $1-2(P<0.0001)$.

The risk of 24-week confirmed disability progression throughout 6 years was substantially reduced among newly diagnosed patients receiving continuous DMF BID treatment (DMF/DMF) compared with those switching from PBO to DMF BID (Fig. 2). Based on Kaplan-Meier estimates, the proportion (95\% CI) of patients with 24-week confirmed disability progression as measured by EDSS was $15.7 \%(10.3 \%, 23.7 \%)$ in DMF/DMF patients and $24.3 \%(15.9 \%, 36.2 \%)$ in the $\mathrm{PBO} / \mathrm{DMF}$ treatment group after 6-year minimum follow-up (hazard ratio: 0.51; 95\% CI: 0.27, 0.97), which represented a $49 \%$ risk reduction for DMF/DMF versus PBO/DMF $(P=0.0397)$. The overall event rate of EDSS progression remains low; therefore, median EDSS scores remained stable over the study period. Specifically, the median EDSS at the end of 6 years remained 2.0, the same as the median EDSS at baseline.

\section{AEs}

In the newly diagnosed population, overall incidence of AEs was similar between $\mathrm{PBO} /$ DMF-treated (94\%) and DMF/DMF-treated patients (92\%; Table 2). AEs reported most frequently in patients receiving continuous DMF BID included MS relapse, nasopharyngitis, upper respiratory tract infection, urinary tract infection, and flushing. The most common AE reported by patients new to DMF treatment in ENDORSE included 
(a) Cummulative ARR of Years 0-6

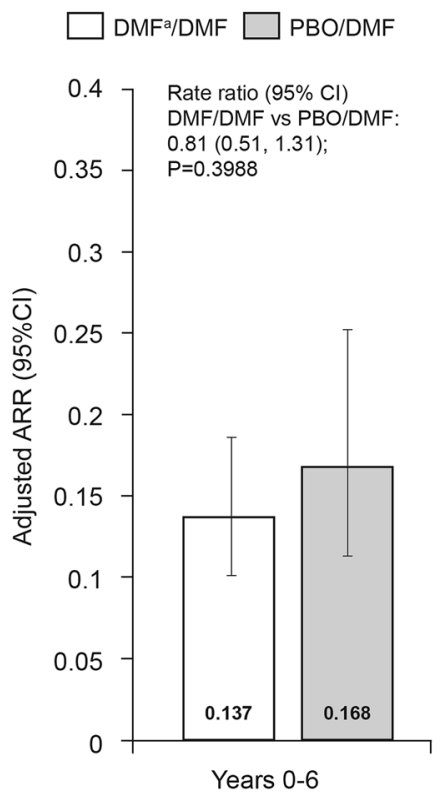

Fig. 1 Cumulative ARR. ARR was calculated using a negative binomial regression model, adjusted for baseline Expanded Disability Status Scale $(\leq 2.0$ vs. $>2.0)$, baseline age ( $<40$ vs. $\geq 40$ years), region, and number of relapses in the 1 year prior to DEFINE/CONFIRM study entry.

flushing, MS relapse, and headache. In the $\mathrm{DMF} / \mathrm{DMF}$ and $\mathrm{PBO} / \mathrm{DMF}$ groups, $9 \%$ and $18 \%$, respectively, discontinued study treatment due to AEs. Rates of discontinuation due to individual AEs in were low $(\leq 2 \%$ for individual AEs in each treatment group).

\section{DISCUSSION}

DMF showed strong and sustained efficacy across a spectrum of clinical outcomes in newly diagnosed patients with RRMS in this post hoc analysis of integrated data from DEFINE, CONFIRM, and ENDORSE patients having a 6-year minimum follow-up. Patients receiving continuous DMF treatment experienced sustained clinical effects on ARR throughout 6 years of follow-up. DMF treatment also resulted in clinical benefits for

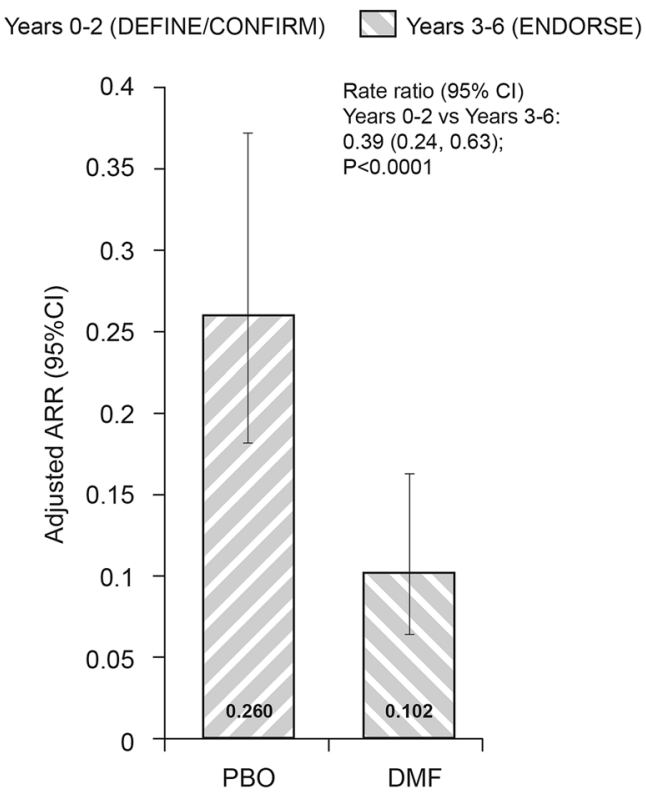

${ }^{a} D M F$ delayed-release dimethyl fumarate (also known as gastro-resistant DMF). ${ }^{\mathrm{b}}$ Based on a repeated negative binomial model for estimated $0-2 / 3-6$ years ARR. ARR annualized relapse rate, $C I$ confidence interval, $P B O$ placebo

patients who switched from $\mathrm{PBO}$ to receiving DMF BID treatment for 4 years, as evidenced by reduced ARR following the switch. Importantly, patients receiving continuous DMF treatment had substantially lower risk for 24-week confirmed disability progression compared with those receiving delayed treatment. This benefit was sustained with 6 years of minimum follow-up.

The effects of DMF in the newly diagnosed population were numerically stronger than those seen in the overall ITT population of ENDORSE [22] and consistent with findings from previous integrated studies of DEFINE and CONFIRM [19]. Although limited, these results support the notion that intervention at the early stages of RRMS may improve treatment outcomes. Indeed, it has been reported that acute exacerbations of MS have a 

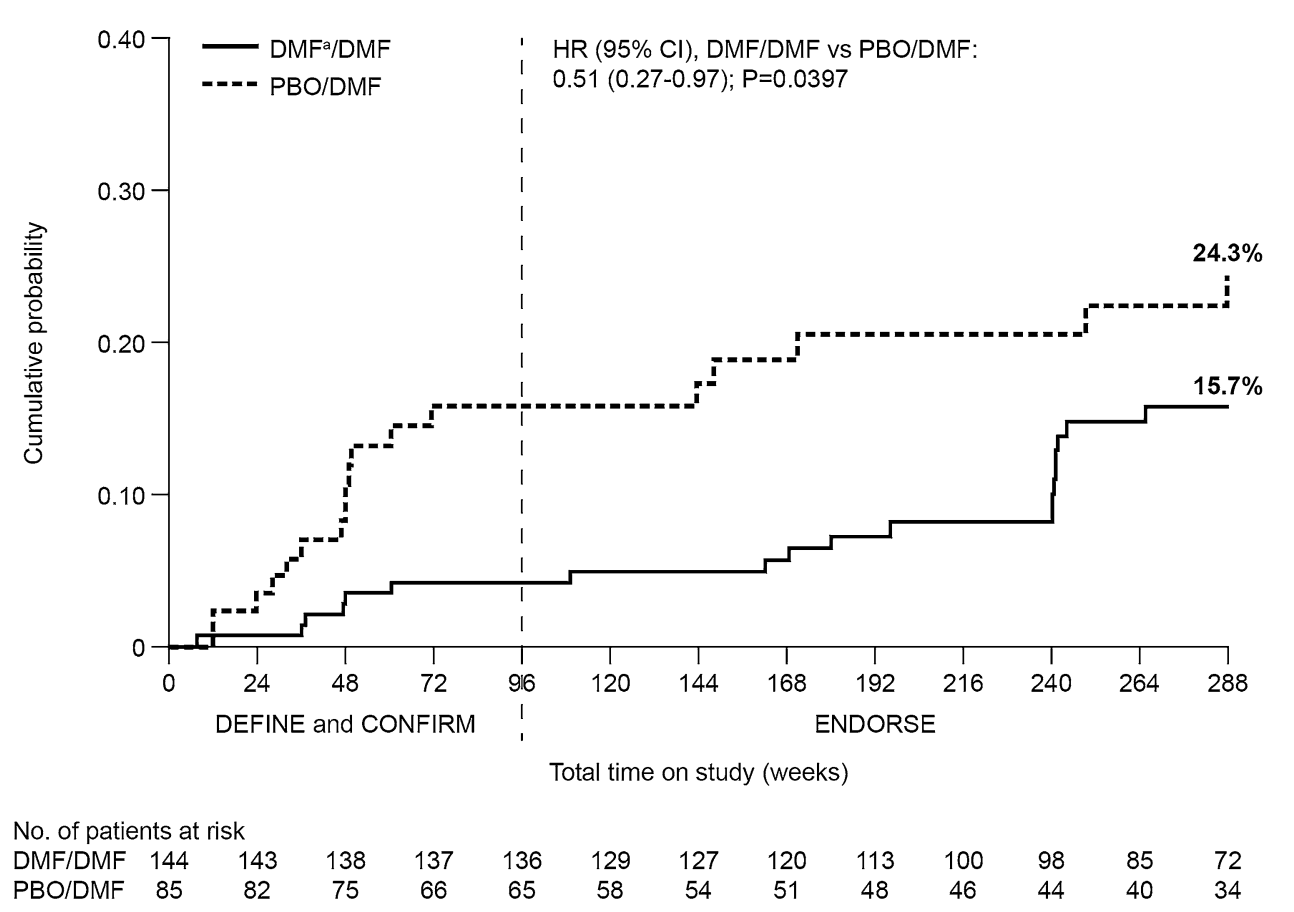

Fig. 2 Proportion of patients with 24-week confirmed disability progression. Confirmed progression of disability is defined as $>1.0$-point increase on EDSS from a baseline EDSS $>1.0$ confirmed for 24 weeks or $>1.5$-point increase on EDSS from a baseline EDSS of 0 confirmed for

sustained effect on accrued impairment in MS [23]. Therefore, decreasing the total number of events experienced in a lifetime may reduce the overall impairment, underlying improved long-term outcomes from earlier treatment.

It should be noted that the newly diagnosed cohort assessed in this analysis was limited by the small sample size and the post hoc nature of the analysis. As with other long-term extension trials [24, 25], bias could also result from the disproportionate discontinuation of patients who experienced suboptimal efficacy or AEs during the ENDORSE extension period, although the impact would be expected to be similar between the two arms, or because not all patients completing DEFINE and CONFIRM chose to enroll in ENDORSE. Therefore, results of the present analysis should be interpreted with caution.
24 weeks. Patients were censored if they withdrew from the study or switched to alternative MS medication without a progression. ${ }^{2} D M F$ delayed-release dimethyl fumarate (also known as gastro-resistant DMF). EDSS Expanded Disability Status Scale, $H R$ hazard ratio, $P B O$ placebo

Access of the central nervous system by autoreactive lymphocytes is thought to trigger a cascade of events that initiate the demyelination, axonal transection, and neurodegeneration associated with RRMS. This is followed by extensive neuronal loss and gliosis in later stages $[7,26]$. Therefore, therapeutic interventions in newly diagnosed patients with RRMS may have the greatest potential to slow the accumulation of damage in the long term. This assertion is supported by findings that long-term outcomes are poorer in patients with a greater frequency of relapse and higher lesion load in early MS [27-29]. This evidence supports the notion that the opportunity for maximal therapeutic effect has an early window, with the association between MS disease activity and long-term clinical prognosis becoming attenuated over time. [28, 
Table 2 Overall incidence of AEs (occurring at an incidence of $\geq 10 \%$ ) in the newly diagnosed population

\begin{tabular}{lcc}
\hline Event & $\mathbf{D M F}^{\mathbf{a}} / \mathbf{D M F}^{\mathbf{a}}(\boldsymbol{n}=\mathbf{1 4 4})$ & $\mathbf{P B O} / \mathbf{D M F} \mathbf{a}^{\mathbf{a}}(\boldsymbol{n}=\mathbf{8 5})$ \\
\hline Any AE, $n$ (\%) & $132(92)$ & $80(94)$ \\
MS relapse & $41(28)$ & $22(26)$ \\
Nasopharyngitis & $39(27)$ & $12(14)$ \\
Flushing & $21(15)$ & $26(31)$ \\
Upper respiratory tract infection & $29(20)$ & $11(13)$ \\
Urinary tract infection & $24(17)$ & $9(11)$ \\
Headache & $20(14)$ & $14(16)$ \\
Diarrhea & $20(14)$ & $11(13)$ \\
Back pain & $19(13)$ & $9(11)$ \\
Fatigue & $13(9)$ & $10(12)$ \\
Upper abdominal pain & $5(3)$ & $9(11)$ \\
Pain in extremity & $15(10)$ & $7(8)$ \\
\hline
\end{tabular}

$A E$ adverse event, $M S$ multiple sclerosis, $P B O$ placebo

${ }^{a} D M F$ delayed-release dimethyl fumarate (also known as gastro-resistant DMF)

29]. Furthermore, MRI and pathological data support MS causing axonal damage even when there are no clinical signs of the disease [30]. Consequently, a number of guidelines, including those issued by the National Multiple Sclerosis Society, recommend early intervention as standard of care [31-33].

Currently, there are no universal criteria for defining 'newly diagnosed'. Newly diagnosed patients have been variously defined using several criteria, either alone or in combination: time from symptom onset or diagnosis, EDSS score, clinical presentation (consistent with CIS), and progression from CIS to CDMS. Time since diagnosis is the most varied of the newly diagnosed criteria; this duration of time has varied from as short as immediately following diagnosis [34] to as long as $8-10$ years after diagnosis $[35,36]$. For the purpose of this study, the newly diagnosed patients were initially described as being diagnosed with RRMS per McDonald criteria
[20] within 1 year from study entry. Inherent in the ENDORSE study design, this analysis assesses newly diagnosed patients treated with DMF at 2 different times from diagnosis with RRMS. DMF/DMF patients received DMF treatment within 1 year of diagnosis. Meanwhile, PBO/DMF patients received DMF treatment within 3 years of diagnosis. It is important to note patients analyzed within this study have already progressed past the CIS and CDMS stages of MS and were either treatment-naïve or previously treated with corticosteroids alone.

Based on this post hoc analysis, DMF demonstrated a safety and tolerability profile in newly diagnosed patients that was comparable with that of the ENDORSE overall safety population [37]. Flushing, nasopharyngitis, and MS relapse were among the most common AEs reported by both DMF/ DMF and $\mathrm{PBO} / \mathrm{DMF}$ newly diagnosed patients. In the overall ENDORSE safety population, the 
most common AEs were MS relapse and nasopharyngitis in the DMF/DMF patients, while flushing and gastrointestinal (GI)-related events were more common among patients previously treated with $\mathrm{PBO}$ and new to DMF treatment [37]. This is not surprising given the well-known observation that flushing and GI-related events tended to be transient and decrease substantially after the first 1-2 months. The incidence of AEs leading to discontinuation were higher in newly diagnosed patients with RRMS who were new to DMF in ENDORSE (PBO/DMF; 18\%) compared with those receiving continuous treatment (DMF/DMF; 9\%). This is consistent with the overall population, in which $17 \%$ of $\mathrm{PBO} / \mathrm{DMF}$ and $7 \%$ DMF/DMF patients discontinued due to AEs. The observation that a higher proportion of patients new to DMF discontinued due to AEs, in both the newly diagnosed and overall population, can largely be explained by the occurrence of flushing and GI events that tend to occur early in therapy with DMF [17, 18, 38]. Rates of discontinuation due to individual GI-related AEs were similar in patients new to DMF in the newly diagnosed cohort compared with the overall population ( $\leq 3 \%$ discontinued due to individual GI-related AEs). Among patients new to DMF, $4 \%$ and $0 \%$ discontinued due to flushing in the overall population and newly diagnosed cohort, respectively. In patients continuing DMF, $\leq 1 \%$ of patients each discontinued due to flushing or GI-related events in both the overall population and the newly diagnosed cohort.

\section{CONCLUSIONS}

After 6 years minimum follow-up in patients who received continuous DMF treatment ( 2 years in DEFINE or CONFIRM, followed by 4 years in ENDORSE), the ARR and the proportion of patients with confirmed disability progression remained low: from Years 0-6, the ARR was 0.137 (95\% CI 0.101, $0.186)$ and the proportion of patients with disability progression was $15.7 \% \quad(95 \%$ CI $10.3 \%, 23.7 \%)$. In patients who switched from PBO (Years 0-2) to DMF (Years 3-6), the ARR was significantly reduced (61\% risk reduction) after switching to DMF. Importantly, patients receiving continuous DMF treatment had substantially lower risk for 24-week confirmed disability progression over the course of 6 years compared with those who received delayed treatment. Together, results of this post hoc analysis show that treatment with DMF results in strong and sustained clinical effects in newly diagnosed patients with RRMS and suggest greater benefit with early initiation of treatment in this patient population. However, results should be interpreted with caution as the sample size was small in the newly diagnosed cohort.

\section{ACKNOWLEDGMENTS}

Biogen funded the DEFINE, CONFIRM, and ENDORSE studies. The article processing charges for this study were funded by Biogen. All named authors meet the International Committee of Medical Journal Editors (ICMJE) criteria for authorship for this manuscript, take responsibility for the integrity of the work as a whole, and have given final approval to the version to be published. All authors had full access to all relevant aggregated study data required to understand and report research findings for this study and take complete responsibility for the integrity of such data and the accuracy of the data analysis. Individual contributions are as follows: study conception and design: RG, GG, JTP, RJF; 
acquisition of data: RG, GG, JTP, RJF; analysis and interpretation: RG, GG, JTP, RJF, AZ, JLM; drafting and review of the manuscript: $R G, G G$, JTP, RJF, AZ, JLM. Biogen reviewed and provided feedback on the paper to the authors. The authors had full editorial control of the paper, and provided their final approval of all content. Biogen provided funding for medical writing support in the development of this paper; Gina Rocco PhD, from Complete Medical Communications (Hackensack, NJ, USA) wrote the first draft of the manuscript based on input from authors, and Elise Chahine from Complete Medical Communications (Hackensack, NJ, USA) copyedited and styled the manuscript per journal requirements. James Xiao (Biogen) provided support for statistical analyses and verified the accuracy of the data.

Disclosures. Ralf Gold has received consultant fees from Bayer HealthCare, Biogen, Genzyme, Merck Serono, Novartis, and Teva Neuroscience; grant/research support from Bayer HealthCare, Biogen, Genzyme, Merck Serono, Novartis, and Teva Neuroscience; compensation from Sage as editor of Therapeutic Advances in Neurological Disorders. Gavin Giovannoni has received honoraria from Abbvie, Bayer HealthCare, Biogen, Canbex Therapeutics, Five Prime Therapeutics, Genzyme, GlaxoSmithKline, GW Pharma, Merck, Merck Serono, Novartis, Protein Discovery Laboratories, Oxford Pharmagenesis, Roche, Synthon, Teva Neuroscience, UCB, and Vertex; research grant support from Biogen, Ironwood, Merck Serono, Merz, and Novartis; compensation from Elsevier as co-chief editor of Multiple Sclerosis and Related Disorders. J. Theodore Phillips has received consultant fees from Acorda, Biogen, Genentech, Genzyme, Merck Serono, Sanofi, and Xenoport. Robert J. Fox has received consultant fees from Actelion, Biogen, MedDay, Novartis, Questcor, Teva, and Xenoport; served on advisory committees for Actelion, Biogen, and Novartis; research grant funding from Novartis. Annie Zhang is an employee of and holds stock/stock options in Biogen. Jing L. Marantz is an employee of and holds stock/stock options in Biogen.

Compliance with Ethics Guidelines. All procedures followed were in accordance with the ethical standards of the responsible committee on human experimentation (institutional and national) and with the Helsinki Declaration of 1964 , as revised in 2013. Informed consent was obtained from all patients for being included in the study.

Open Access. This article is distributed under the terms of the Creative Commons Attribution-NonCommercial 4.0 International License (http://creativecommons.org/licenses/ by-nc/4.0/), which permits any noncommercial use, distribution, and reproduction in any medium, provided you give appropriate credit to the original author(s) and the source, provide a link to the Creative Commons license, and indicate if changes were made.

\section{REFERENCES}

1. Gilgun-Sherki Y, Melamed E, Offen D. The role of oxidative stress in the pathogenesis of multiple sclerosis: the need for effective antioxidant therapy. J Neurol. 2004;251:261-8.

2. Noseworthy JH, Lucchinetti C, Rodriguez M, Weinshenker BG. Multiple sclerosis. N Engl J Med. 2000;343:938-52.

3. Luessi F, Siffrin V, Zipp F. Neurodegeneration in multiple sclerosis: novel treatment strategies. Expert Rev Neurother. 2012;12:1061-76.

4. Zwibel HL, Smrtka J. Improving quality of life in multiple sclerosis: an unmet need. Am J Manag Care. 2011;17(Suppl 5 Improving):S139-45. 
5. Lublin FD. Clinical features and diagnosis of multiple sclerosis. Neurol Clin. 2005;23:1-15, v.

6. Lublin FD, Reingold SC. Defining the clinical course of multiple sclerosis: results of an international survey. National Multiple Sclerosis Society (USA) Advisory Committee on Clinical Trials of New Agents in Multiple Sclerosis. Neurology. 1996;46:907-11.

7. Compston A, Coles A. Multiple sclerosis. Lancet. 2008;372:1502-17.

8. Stone LA, Smith ME, Albert PS, et al. Blood-brain barrier disruption on contrast-enhanced MRI in patients with mild relapsing-remitting multiple sclerosis: relationship to course, gender, and age. Neurology. 1995;45:1122-6.

9. Comi G, Filippi M, Barkhof F et al. Effect of early interferon treatment on conversion to definite multiple sclerosis: a randomised study. Lancet. 2001;357:1576-82.

10. Comi G, Martinelli V, Rodegher M, et al. Effect of glatiramer acetate on conversion to clinically definite multiple sclerosis in patients with clinically isolated syndrome (PreCISe study): a randomised, double-blind, placebo-controlled trial. Lancet. 2009;374:1503-11.

11. Comi G, De SN, Freedman MS, et al. Comparison of two dosing frequencies of subcutaneous interferon beta-1a in patients with a first clinical demyelinating event suggestive of multiple sclerosis (REFLEX): a phase 3 randomised controlled trial. Lancet Neurol. 2012;11:33-41.

12. Jacobs LD, Beck RW, Simon JH, et al. Intramuscular interferon beta-1a therapy initiated during a first demyelinating event in multiple sclerosis. CHAMPS Study Group. N Engl J Med. 2000;343:898-904.

13. Kappos L, Polman CH, Freedman MS, et al. Treatment with interferon beta-1b delays conversion to clinically definite and McDonald MS in patients with clinically isolated syndromes. Neurology. 2006;67:1242-9.

14. Kappos L, Freedman MS, Polman $\mathrm{CH}$, et al. Long-term effect of early treatment with interferon beta-1b after a first clinical event suggestive of multiple sclerosis: 5-year active treatment extension of the phase 3 BENEFIT trial. Lancet Neurol. 2009;8:987-97.

15. Kappos L, Freedman MS, Polman CH, et al. Effect of early versus delayed interferon beta- $1 \mathrm{~b}$ treatment on disability after a first clinical event suggestive of multiple sclerosis: a 3-year follow-up analysis of the BENEFIT study. Lancet. 2007;370:389-97.
16. O'Connor P, Kinkel RP, Kremenchutzky M. Efficacy of intramuscular interferon beta-1a in patients with clinically isolated syndrome: analysis of subgroups based on new risk criteria. Mult Scler. 2009;15:728-34.

17. Fox RJ, Miller DH, Phillips JT, et al. Placebo-controlled phase 3 study of oral BG-12 or glatiramer in multiple sclerosis. $\mathrm{N}$ Engl J Med. 2012;367:1087-97.

18. Gold R, Kappos L, Arnold DL, et al. Placebo-controlled phase 3 study of oral BG-12 for relapsing multiple sclerosis. $\mathrm{N}$ Engl $\mathrm{J}$ Med. 2012;367:1098-107.

19. Gold R, Giovannoni G, Phillips JT, et al. Efficacy and safety of delayed-release dimethyl fumarate in patients newly diagnosed with relapsing-remitting multiple sclerosis (RRMS). Mult Scler. 2015;21:57-66.

20. Polman $\mathrm{CH}$, Reingold SC, Edan G, et al. Diagnostic criteria for multiple sclerosis: 2005 revisions to the "McDonald Criteria". Ann Neurol. 2005;58:840-6.

21. Kurtzke JF. Rating neurologic impairment in multiple sclerosis: an expanded disability status scale (EDSS). Neurology. 1983;33:1444-52.

22. Hutchinson M, Gold R, Fox RJ, et al. Six-year follow-up of delayed-release dimethyl fumarate in RRMS: integrated clinical efficacy data from the DEFINE, CONFIRM, and ENDORSE study. Mult Scler. 2015;23(S11):246.

23. Lublin FD, Baier M, Cutter G. Effect of relapses on development of residual deficit in multiple sclerosis. Neurology. 2003;61:1528-32.

24. Kappos L, O'Connor P, Radue EW, et al. Long-term effects of fingolimod in multiple sclerosis: the randomized FREEDOMS extension trial. Neurology. 2015;84:1582-91.

25. Khatri B, Barkhof F, Comi G, et al. Comparison of fingolimod with interferon beta-1a in relapsing-remitting multiple sclerosis: a randomised extension of the TRANSFORMS study. Lancet Neurol. 2011;10:520-9.

26. Trapp BD, Peterson J, Ransohoff RM, Rudick R, Mork S, Bo L. Axonal transection in the lesions of multiple sclerosis. N Engl J Med. 1998;338:278-85.

27. Brex PA, Ciccarelli O, O'Riordan JI, Sailer M, Thompson AJ, Miller DH. A longitudinal study of abnormalities on MRI and disability from multiple sclerosis. N Engl J Med. 2002;346:158-64. 
28. Confavreux C, Vukusic S, Adeleine P. Early clinical predictors and progression of irreversible disability in multiple sclerosis: an amnesic process. Brain. 2003;126:770-82.

29. Scalfari A, Neuhaus A, Degenhardt A, et al. The natural history of multiple sclerosis: a geographically based study 10: relapses and long-term disability. Brain. 2010;133:1914-29.

30. McFarland HF. The lesion in multiple sclerosis: clinical, pathological, and magnetic resonance imaging considerations. J Neurol Neurosurg Psychiatry. 1998;64(Suppl 1):S26-30.

31. Goodin DS, Frohman EM, Garmany GP Jr, et al. Disease modifying therapies in multiple sclerosis: report of the Therapeutics and Technology Assessment Subcommittee of the American Academy of Neurology and the MS Council for Clinical Practice Guidelines. Neurology. 2002;58:169-78.

32. Multiple Sclerosis Coalition. The use of disease-modifying therapies in multiple sclerosis. 2015. http://ms-coalition.org/cms/images/stories/ DMTfullpaper_2015update_final.pdff. Accessed 12 Nov 2015.

33. Goodin DS, Bates D. Treatment of early multiple sclerosis: the value of treatment initiation after a first clinical episode. Mult Scler. 2009;15:1175-82.
34. Bellmann-Strobl J, Stiepani H, Wuerfel J, et al. MR spectroscopy (MRS) and magnetisation transfer imaging (MTI), lesion load and clinical scores in early relapsing remitting multiple sclerosis: a combined cross-sectional and longitudinal study. Eur Radiol. 2009;19:2066-74.

35. Dennison L, Moss-Morris R, Silber E, Galea I, Chalder T. Cognitive and behavioural correlates of different domains of psychological adjustment in early-stage multiple sclerosis. J Psychosom Res. 2010;69:353-61.

36. Dennison L, Yardley L, Devereux A, Moss-Morris R. Experiences of adjusting to early stage Multiple Sclerosis. J Health Psychol. 2011;16:478-88.

37. Pozzilli C, Phillips JT, Fox RJ, et al. Long-term follow-up of the safety of delayed-release dimethyl fumarate in RRMS: interim results from the ENDORSE extension study. Mult Scler. 2015;23(S11):247.

38. Phillips JT, Hutchinson M, Fox R, Gold R, Havrdova E. Managing flushing and gastrointestinal events associated with delayed-release dimethyl fumarate: experiences of an international panel. Mult Scler Relat Disord. 2014;3:513-9. 